$10,11,05$

\title{
Влияние магнитного поля на фазовые переходы в модели Гейзенберга на треугольной решетке
}

\author{
(C) А.К. Муртазаев, М.К. Бадиев, М.К. Рамазанов, М.А. Магомедов \\ Институт фозики ДФИЦ РАН, \\ Махачкала, Россия \\ E-mail: m_zagir@mail.ru
}

Поступила в Редакцию 29 марта 2021 г.

В окончательной редакции 2 апреля 2021 г.

Принята к публикации 3 апреля 2021 г.

\begin{abstract}
Методом Монте-Карло выполнены исследования фазовых переходов, магнитных и термодинамических свойств трехмерной антиферромагнитной модели Гейзенберга на слоистой треугольной решетке в магнитном поле. Исследования проведены в интервале изменения величины магнитного поля $0 \leq h \leq 12$. Получены магнитные структуры основного состояния в широком интервале значений магнитного поля. На основе гистограммного метода анализа данных определен характер фазовых переходов. Установлено, что в интервале $0 \leq h \leq 10$ реализуется фазовый переход первого рода. Показано, что дальнейшее увеличение величины магнитного поля снимает вырождение основного состояния и размывает фазовый переход.
\end{abstract}

Ключевые слова: фрустрации, метод Монте-Карло, модель Гейзенберга, фазовый переход.

DOI: $10.21883 /$ FTT.2021.08.51168.068

\section{1. Введение}

В последние десятилетия значительное внимание уделяется исследованию магнитных структур, фазовых переходов (ФП), магнитных, термодинамических и критических свойств в спиновых системах с фрустрациями. Это связано с тем, что такие системы проявляют поведение, существенно отличное от соответствующих нефрустрированных систем. Причина такого поведения заключается в сильном вырождении в спиновой подсистеме, эффективном ослаблении связи, и, как следствие, высокой чувствительности к различным возмущающим факторам. В настоящее время вопрос о влиянии возмущений различной природы, таких как анизотропия, взаимодействия следующих за ближайшими соседей, внешнее магнитное поле, тепловые и квантовые флуктуации, немагнитные примеси, дефекты, деформации и др. на фрустрированные магнитные системы имеет принципиальное значение [1-10]. Включение этих факторов приводит к большому разнообразию фаз и ФП в таких магнетиках. Результаты экспериментальных [11] и теоретических [12-14] исследований показывают, что наличие фрустраций приводит к существенному изменению ряда свойств фундаментального характера. Особый интерес представляют проблемы, связанные с определением характера ФП, с особенностями и факторами, влияющими на формирование классов универсальности магнитного и кирального критического поведения.

Одним из наиболее интенсивно исследуемых фрустрированных моделей является антиферромагнитная модель Гейзенберга на слоистой треугольной решетке. К настоящему моменту времени, эта модель достаточно хорошо изучена как для квантовых, так и для классических систем [15-20]. Однако остаются невыясненными неко- торые вопросы, связанные с влиянием внешнего магнитного поля на ФП, термодинамические, критические и магнитные свойства слоистых антиферромагнетиков с треугольной геометрией.

С одной стороны, экспериментальные, численные, а также теоретические данные, полученные методом ренормгрупп на основе $\varepsilon$-разложения, представленные в работах [12-16,19,21-25], свидетельствуют о наличии ФП второго рода и принадлежности антиферромагнитной модели Гейзенберга на треугольной решетке к новому киральному классу универсальности критического поведения. С другой стороны, имеются данные, полученные с использованием непертурбативного подхода метода ренормгрупп, которые показывают наличие в этой модели ФП первого рода, а также слабо выраженного ФП первого рода, близкого к ФП второго рода с эффективными критическими индексами [26-29].

Исследование влияния внешнего магнитного поля на ФП в модели Изинга на слоистой треугольной решетке показало, что в интервале значения $0 \leq h \leq 6$ реализуется ФП второго рода [30]. Обнаружено, что дальнейшее увеличение величины магнитного поля снимает вырождение основного состояния и размывает ФП. В работе [8] показано, что в двумерной модели Гейзенберга в области малых магнитных полей при $h=2$ параметры порядка для симметрий $Z_{3}$ и $\mathrm{O}(2)$ демонстрируют поведение, типичное для перехода второго рода и КостерлицаТаулеса (KT) перехода соответственно, с различными температурами перехода. В области сильного поля, где $3 \leq h \leq 9$ параметр порядка для симметрии $Z_{3}$ демонстрирует поведение перехода второго рода, в то время как другой параметр порядка, связанный с компонентами, перпендикулярными полю, демонстрирует, что время релаксации расходится с асимптотической 
формой, указывая на переход второго рода, а не переход КТ. Результаты, полученные для квазидвумерной модели Гейзенберга на треугольной решетке с помощью моделирования методом Монте-Карло (МК) в нулевом магнитном поле показывают наличие трех переходов Березинского-Костерлица-Таулеса с конечной температурой [31]. Первые два перехода связаны с нарушением дискретной группы симметрии $Z_{6}$, а третий переход связан с квазидальним упорядочением поперечных компонент. Обнаружено, что магнитное поле, приложенное параллельно оси анизотропии, снижает дискретную симметрию до $Z_{3}$.

Результаты исследований модели Гейзенберга в магнитном поле полученные с использованием теории среднего поля и численного метода приведены в работах [32-34]. В работе [32] представлена фазовая диаграмма Гейзенберговского антиферромагнетика на треугольной решетке с взаимодействиями ближайших $\left(J_{1}\right)$ и следующих ближайших $\left(J_{2}\right)$ соседей в магнитном поле. Показано, что классическая модель Гейзенберга имеет случайное вырождение для всех соотношений $J_{2} / J_{1}$. Обнаружено, что вырождение снимается квантовыми флуктуациями. В работе [34] обнаружено, что квантовый механизм „порядок-беспорядок“ стабилизирует три различные фазы в зависимости от напряженности поля, пока система не достигнет магнитного насыщения. При более сильных полях авторы обнаружили неожиданную нематическую природу, обусловленную нетривиальным частичным нарушением симметрии. Кроме того, обнаружено, что сильная нематическая природа низкополевой и высокополевой фаз приводит к возникновению дробных вихрей и антивихрей.

Анализ литературных данных показывает, что внешнее магнитное поле влияет на многие физические свойства спиновых систем. Поэтому в данной работе нами изучается влияние магнитного поля на характер ФП, магнитные и термодинамические свойства антиферромагнитной модели Гейзенберга на слоистой треугольной решетке.

Интерес к этой модели обусловлен тем, что влияние внешнего магнитного поля на ФП, магнитные и термодинамические свойства этой модели на основе современных методов до сих пор никем не проводилось. Поэтому, исследование трехмерной антиферромагнитной модели Гейзенберга на слоистой треугольной решетке в магнитном поле на основе современных методов и идей позволит получить ответ на ряд вопросов, связанных с ФП, магнитными и термодинамическими свойствами фрустрированных спиновых систем, а также влияния на них внешнего магнитного поля.

\section{2. Модель и метод исследования}

Гамильтониан антиферромагнитной модели Гейзенберга на слоистой треугольной решетке может быть представлен в следующем виде [16]:

$$
H=-J \sum_{\langle i, j\rangle}\left(\mathbf{S}_{i} \cdot \mathbf{S}_{j}\right)-h \sum_{i} \mathbf{S}_{i}^{z},
$$

где $\mathbf{S}_{i}-$ трехкомпонентный единичный вектор $\mathbf{S}_{i}=\left(S_{i}^{x}, S_{i}^{y}, S_{i}^{z}\right), J<0-$ константа антиферромагнитного обменного взаимодействия. Решетка состоит из двумерных треугольных слоев, сложенных по ортогональной оси. Первый член в формуле (1) характеризует антиферромагнитное взаимодействие всех ближайших соседей, $h$ - внешнее магнитное поле. Величина значений внешнего магнитного поля менялась в интервале $0.0 \leq h \leq 12.0$. Все параметры приводятся в безразмерных величинах.

В настоящее время спиновые системы с фрустрациями на основе микроскопических гамильтонианов успешно изучаются на основе метода МК [35-39]. В последнее время разработано много новых вариантов алгоритмов метода МК. Одними из наиболее эффективных для исследования подобных систем являются репличный обменный алгоритм [40].

Репличный обменный алгоритм был использован нами в следующем виде:

1. Одновременно моделируются $N$ реплик $X_{1}$, $X_{2}, \ldots X_{N}$ с температурами $T_{1}, T_{2}, \ldots T_{N}$.

2. После выполнения одного МК-шага/спин для всех реплик производится обмен данными между парой соседних реплик $X_{i}$ и $X_{i+1}$ в соответствии со схемой Метрополиса с вероятностью

$$
w\left(X_{i} \rightarrow X_{i+1}\right)= \begin{cases}1, & \text { for } \Delta \leq 0 \\ \exp (-\Delta), & \text { for } \Delta>0\end{cases}
$$

где $\Delta=\left(U_{i}-U_{i+1}\right) \cdot\left(1 / T_{i}-1 / T_{i+1}\right), U_{i}$ и $U_{i+1}-$ внутренние энергии реплик. Преимущество этого метода в том, что вероятность обмена априори известна, тогда как для других алгоритмов определение вероятности очень утомительно и отнимает много времени.

Для анализа характера ФП нами был использован гистограммный метод анализа данных метода МК $[41,42]$. Расчеты проводились для систем с периодическими граничными условиями и линейными размерами $L \times L \times L=N, L=12 \div 90$, где $L$ измеряется в размерах элементарной ячейки.

\section{3. Результаты моделирования}

На рис. 1 показана магнитная структура основного состояния исследуемой модели, полученная нами для соседних слоев. Структура упорядоченной фазы образована чередованием структур, показанных на рис. 1.

На рис. 2 приведены спиновые структуры основного состояния для разных значений величины магнитного поля при нулевой температуре. Из рисунка видно, что при $h=0$ в основном состоянии система принимает $120^{\circ}$ структуру. При включении внешнего магнитного 


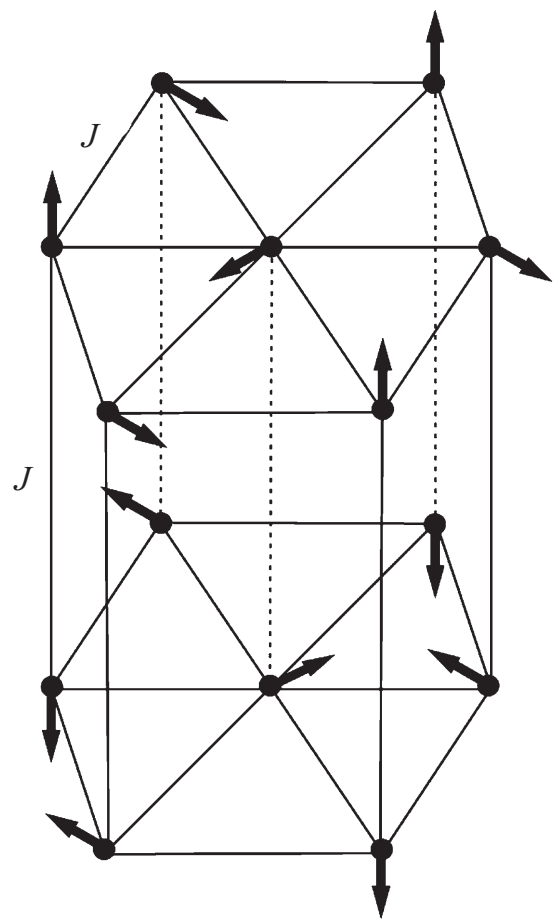

Рис. 1. Магнитная структура основного состояния.

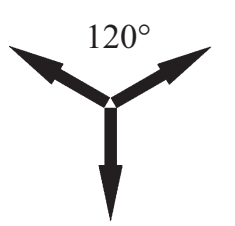

$h=0$

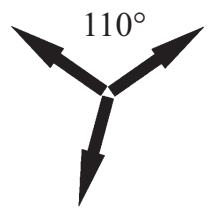

$h=4$

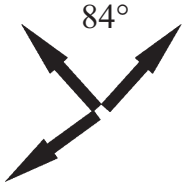

$h=6$

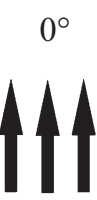

$h=12$
Рис. 2. Спиновая структура основного состояния при нулевой температуре.

поля, спины магнитных подрешеток поворачиваются на определенный угол по направлению поля. С увеличением величины поля угол между спинами и направлением магнитного поля уменьшается. Когда величина магнитного поля $h \geq 12$ угол между спинами становится нулевым. Похожие структуры были обнаружены и в работе [32].

Для наблюдения за температурным ходом поведения теплоемкости $C$ и восприимчивости $\chi$ нами использовались выражения [24,25]:

$$
\begin{gathered}
C=\left(N K^{2}\right)\left(\left\langle U^{2}\right\rangle-\langle U\rangle^{2}\right), \\
\chi= \begin{cases}(N K)\left(\left\langle m^{2}\right\rangle-\langle|m|\rangle^{2}\right), & T<T_{N} \\
(N K)\left\langle m^{2}\right\rangle, & T \geq T_{N}\end{cases}
\end{gathered}
$$

где $K=|J| / k_{B} T, N-$ число частиц, $U-$ внутренняя энергия, $m-$ намагниченность.

Намагниченность системы вычислялось по формуле

$$
m=\frac{3}{N} \sqrt{\left\langle M_{x}^{2}+M_{y}^{2}+M_{z}^{2}\right\rangle / 3},
$$

Намагниченность подрешетки определялась следующим образом [13]:

$$
\left\langle\left|\mathbf{M}_{r}\right|\right\rangle=\left\langle\sqrt{S_{x}^{2}+S_{y}^{2}+S_{z}^{2}}\right\rangle, \quad r=a, b, c .
$$

Параметр порядка системы $q$ вычислялось по формуле

$$
q=\frac{3}{N} \sqrt{\left\langle M_{a}^{2}+M_{b}^{2}+M_{c}^{2}\right\rangle / 3}
$$

где $M_{a}, M_{b}$ и $M_{c}-$ намагниченности трех подрешеток.

На рис. 3 и 4 представлены температурные зависимости теплоемкости $C$ и восприимчивости $\chi$ полученные для системы с линейными размерами $L=36$ при различных значениях величины магнитного поля $h$ (здесь и далее статистическая погрешность не превышает размеров символов, использованных для построения зависимостей). На этих рисунках мы наблюдаем, что в интервале $0 \leq h \leq 10$ вблизи критической температуры наблюдается хорошо выраженный максимум. Отметим, что рост величины магнитного поля $h$ сопровождается с уменьшением и сдвигом максимумов в сторону

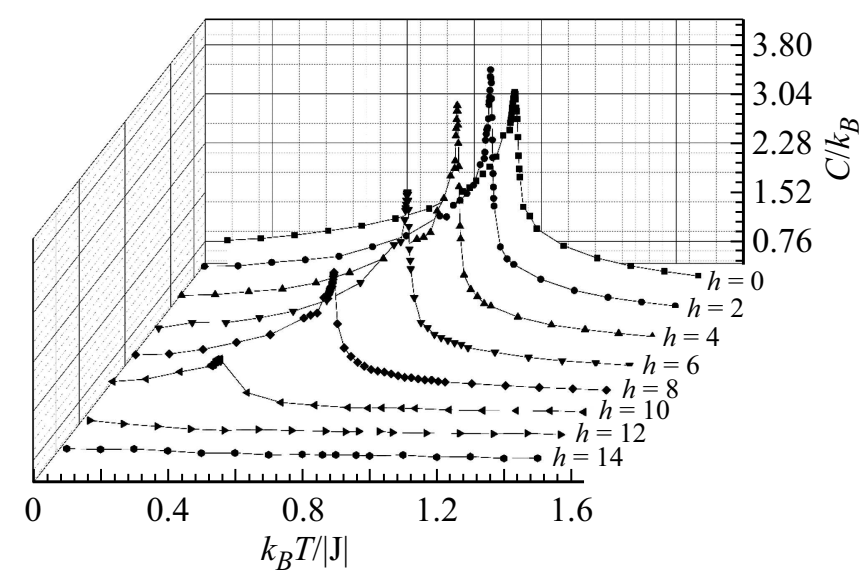

Рис. 3. Зависимость теплоемкости $C / k_{B}$ от температуры при разных значениях $h$.

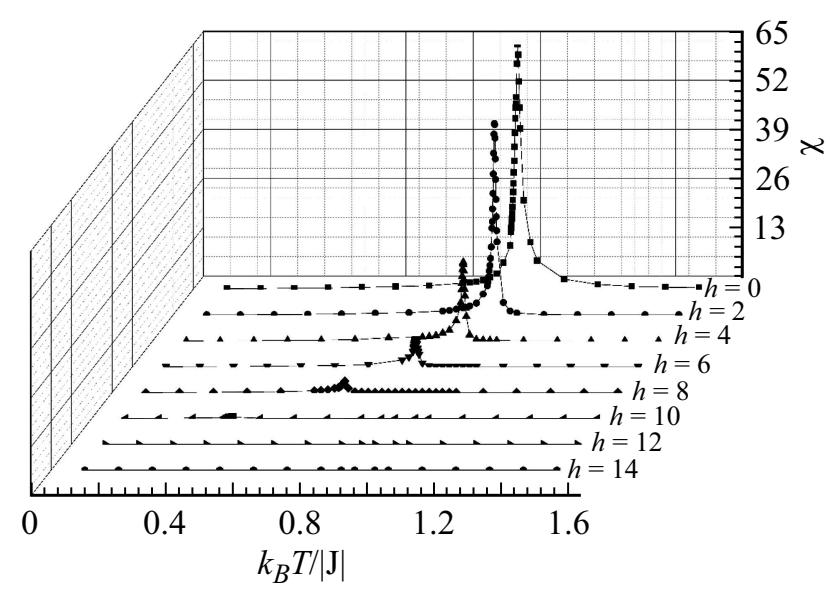

Рис. 4. Зависимость восприимчивости $\chi$ от температуры при разных значениях $h$. 


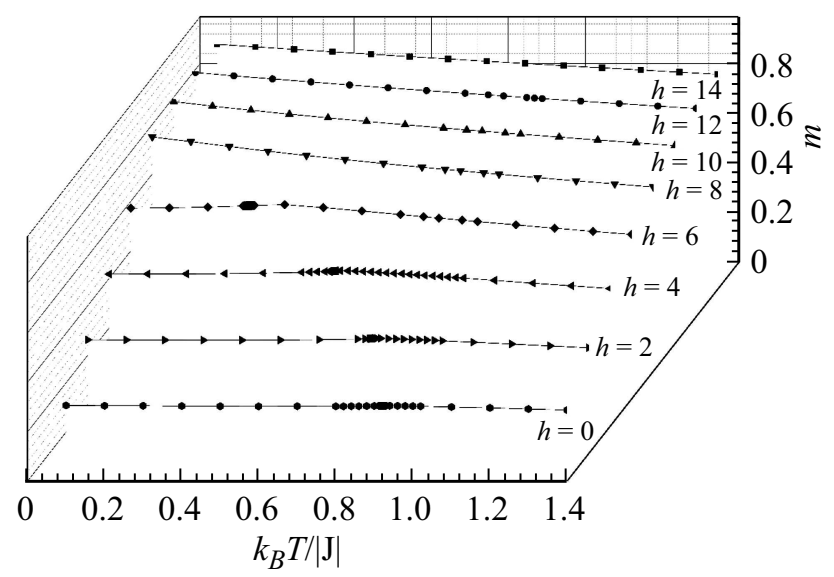

Рис. 5. Зависимость намагниченности $m$ от температуры при разных значениях $h$.

низких температур. Физическая картина происходящего объясняется тем, что увеличение величины магнитного поля приводит быстрому упорядочению системы, уменьшению флуктуаций и соответственно уменьшается температура ФП. При значениях магнитного поля $h \geq 10$ максимумы теплоемкости и восприимчивости становятся более плавными или вовсе исчезают. Это обусловлено тем, что сильное магнитное поле подавляют флуктуации в системе и ФП в системе размывается.

На рис. 5 представлены графики зависимости намагниченности $m$ от температуры для разных значений величины магнитного поля. При отсутствии внешнего магнитного поля, в системе отсутствует порядок, и мы видим значение параметра порядка очень близкое к нулю. При включении поля в системе наблюдается частичное упорядочение и намагниченность в низкотемпературной области имеет отличные от нуля значения. В рассматриваемой модели магнитные подрешетки обладают разной жесткостью по отношению к внешнему магнитному полю. При высоких полях, когда $h>10$ система упорядочивается уже при низких температурах.

На рис. 6 приведены характерные зависимости магнитного параметра порядка $q$ от температуры для различных значений поля $h$. На рисунке видно, что в слабых магнитных полях $(h<8)$ с увеличением магнитного поля в области критической температуры наблюдается резкий спад параметра порядка и смещение в сторону более низких температур. Вместе с этим можно увидеть, что параметр порядка с увеличением $h$ в высокотемпературной области не падает до нуля, как в случае при $h=0$. Это объясняется тем, что магнитное поле выстраивает спины магнитных подрешеток вдоль своего направления и в системе сохраняется остаточный порядок. При более сильных магнитных полях $h>10$, мы замечаем, что система упорядочивается вдоль магнитного поля уже при нулевой температуре и ФП в системе не наблюдается.
Для изучения рода ФП нами использовался гистограммный метод анализа данных метода МК. Этот метод позволяет надежно определить род ФП. Результаты нашей работы показывают, что ФП для значений поля в интервале $0 \leq h \leq 10$ являются переходами первого рода. Это продемонстрировано на рис. 7, где представлены гистограммы распределения энергии для системы с линейными размерами $L=30$ и 90 для $h=0$. График построен для температуры близкой к критической температуре. На рисунке видно, что в зависимости вероятности $P(U)$ от энергии $U$ для системы $L=90$ наблюдаются два хорошо выраженных максимума, которые отсутствуют для системы $L=30$. Такое поведение характерно для ФП перехода первого рода близкому к ФП второго рода. Наличие двойного максимума на энергетической гистограмме является достаточным условием для ФП первого рода. Аналогичное поведение наблюдается для значений поля в интервале $0 \leq h \leq 10$.

Таким образом, ФП в интервале полей $0 \leq h \leq 10$ являются переходами первого рода. Значения критиче-

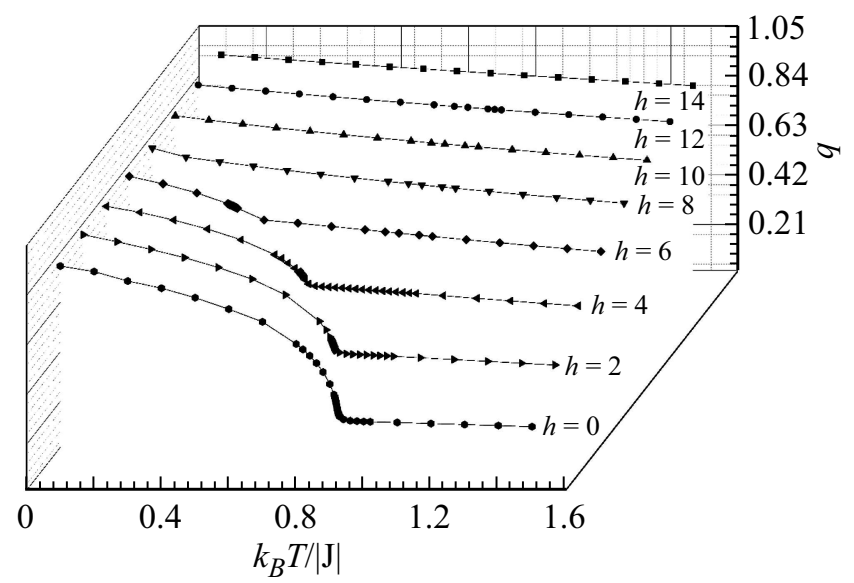

Рис. 6. Зависимость параметра порядка $q$ от температуры при разных значениях $h$.

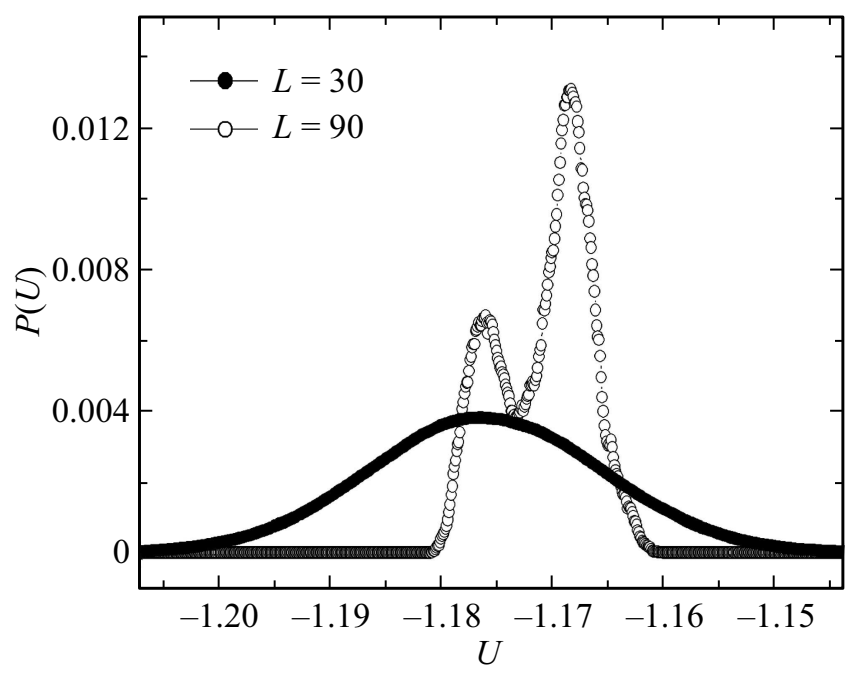

Рис. 7. Гистограмма распределения энергии для $h=0$. 
Значения критичских температур для модели Гейзенберга на треугольной решетке в магнитном поле

\begin{tabular}{c|c|c|c|c|c|c}
\hline$h$ & 0 & 2 & 4 & 6 & 8 & 10 \\
\hline$T_{N}$ & $0.955(4)$ & $0.952(4)$ & $0.920(4)$ & $0.841(4)$ & $0.692(4)$ & $0.411(4)$
\end{tabular}

ских температур в интервале $0 \leq h \leq 10$ приведены в таблице Видно, что с увеличением магнитного поля температура фазового перехода смещается в сторону низких температур.

\section{4. Заключение}

Исследование фазовых переходов, магнитных и термодинамических свойств трехмерной антиферромагнитной модели Гейзенберга на слоистой треугольной решетке в магнитном поле выполнено с использованием репличного алгоритма метода Монте-Карло. На основе гистограммного метода проведен анализ характера фазовых переходов. Установлено, что в исследуемой модели в интервале $0 \leq h \leq 10$ реализуется фазовый переход первого рода. Показано, что дальнейшее увеличение величины магнитного поля приводит к упорядочиванию спинов вдоль магнитного поля и размывает фазовый переход в системе.

\section{Финансирование работы}

Исследование выполнено при финансовой поддержке РФФИ в рамках научных проектов № 19-02-00153-а.

\section{Конфликт интересов}

Авторы заявляют, что у них нет конфликта интересов.

\section{Список литературы}

[1] V.S. Dotsenko. Usp. Fiz. Nauk 165, 481 (1995).

[2] S.E. Korshunov. Usp. Fiz. Nauk 176, 233 (2006).

[3] A. Malakis, P. Kalozoumis, N. Tyraskis. Eur. Phys. J. B 50, 63 (2006).

[4] S.S. Sosin, L.A. Prozorova, A.I. Smirnov. Usp. Fiz. Nauk 175, $92(2005)$

[5] A.Z. Patashinskii, V.L. Pokrovskii. Fluctuation Theory of Phase Transitions Pergamon, Oxford (1979).

[6] Sh.Ma. Modern. Theory of Critical Phenomena SpringerVerlag, London (1976).

[7] Л.Е. Свистов, А.И. Смирнов, Л.А. Прозорова, О.А. Петренко, Ф.Я. Шапиро, Л.Н. Демьянцев. Письма в ЖЭТФ 80, 231 (2004).

[8] M. Kazuaki, O. Yukiyasu. Phys. Rev. B 101, 184427 (2020).

[9] R. Masrour, A. Jabar. Physica A 541, 123377 (2020).

[10] R. Masrour, A. Jabar. Physica A 491, 926 (2018).

[11] С.В. Малеев. УФН 172, 617 (2002).

[12] M. Tisser, B. Delamotte, D. Mouhanna. Phys. Rev. Lett. 84, 5208 (2000).
[13] P. Calabrese, P. Parruccini, A. Pelissetto, E. Vicari. Phys. Rev. B 70, 174439-1 (2004).

[14] G. Zumbach. Nucl. Phys. B 413, 771 (1994).

[15] H. Kawamura. J. Phys. Soc. Jpn. 61, 1299 (1992).

[16] H. Kawamura, S. Miyashita. J. Phys. Soc. Jpn. 54, 4530 (1985).

[17] M. Gvozdikova, P. Melchy, M. Zhitomirsky. J. Phys.: Condens. Matter 23, 164209 (2011).

[18] H. Kawamura, M. Kikuchi. Phys. Rev. B 47, 1134 (1993).

[19] A.V. Chubukov, D.I. Golosov. J. Phys.: Condens. Matter 3, 69 (1991).

[20] H. Kawamura, A. Yamamoto, T. Okubo. J. Phys. Soc. Jpn. 79, 023701 (2010).

[21] A. Peles, B.W. Southern. Phys. Rev. B 67, 184407 (2003).

[22] D. Loison, H.T. Diep. Phys. Rev. B 50, 16453 (1994).

[23] А.К. Муртазаев, М.К. Рамазанов, М.К. Бадиев. ЖЭТФ 132, 1152 (2007).

[24] A.K. Murtazaev, M.K. Ramazanov. Phys. Rev. B 76, 174421 (2007).

[25] А.К. Муртазаев, М.К. Рамазанов, М.К. Бадиев. ФТТ 52, 1157 (2010).

[26] S.A. Antonenko, A.I. Sokolov, V.B. Varnashev. Phys. Lett. A 208, 161 (1995).

[27] A. Peles, B.W. Southern, B. Delamotte, D. Mouhanna, M. Tisser. Phys. Rev. B 69, 220408 (2004).

[28] M. Itakura. J. Phys. Soc. Jpn. 72, 74 (2003).

[29] V. Thanh Ngo, H.T. Diep. Phys. Rev. E 78, 031119 (2008).

[30] A.K. Murtazaev, M.K. Badiev, M.K. Ramazanov, M.A. Magomedov. Physica A 555, 124530 (2020).

[31] P.-E. Melchy, M.E. Zhitomirsky. arXiv: 0812.3574v1 [cond-mat. Str-e1], Dec. (2008).

[32] Ye Mengxing, A.V. Chubukov. Phys. Rev. B 95, 014425 (2017).

[33] Shun-Qing Shen, F.C. Zhang. Phys. Rev. B 66, 172407 (2002).

[34] D. Yamamoto, C. Suzuki, G. Marmorini, S. Okazaki, N. Furukawa. Phys. Rev. Lett. 125, 057204 (2020).

[35] A.K. Murtazaev, M.K. Ramazanov, M.K. Badiev. Physica A 507, 210 (2018).

[36] А.К. Муртазаев, М.К. Рамазанов, К.Ш. Муртазаев, М.А. Магомедов, М.К. Бадиев. ФТТ 62, 229 (2020).

[37] A.K. Murtazaev, D.R. Kurbanova, M.K. Ramazanov. Physica A 545, 123548 (2020).

[38] R. Masrour, A. Jabar, A. Benyoussef, M. Hamedoun. J. Magn. Magn. Mater. 401, 695 (2016).

[39] M.K. Ramazanov, A.K. Murtazaev, M.A. Magomedov. Physica A 521, 543 (2019).

[40] A. Mitsutake, Y. Sugita, Y. Okamoto. Biopolymers (Peptide Sci.) 60, 96 (2001).

[41] F. Wang, D.P. Landau. Phys. Rev. E 64, 056101-1 (2001).

[42] F. Wang, D.P. Landau. Phys. Rev. Lett. 86, 2050 (2001).

Редактор Т.Н. Василевская 